\title{
A systematic DNN-based QSPR modeling methodology for rapid and reliable prediction on flashpoints of chemicals
}

\author{
Huaqiang Wen ${ }^{1}$, Yang $\mathrm{Su}^{2}$, Zihao Wang ${ }^{3}$, saimeng Jin ${ }^{1}$, Jingzheng Ren ${ }^{4}$, Weifeng Shen ${ }^{1}$, \\ and Mario Eden ${ }^{5}$ \\ ${ }^{1}$ Chongqing University \\ ${ }^{2}$ Chongqing University of Science and Technology \\ ${ }^{3}$ Max Planck Institute for Dynamics of Complex Technical Systems \\ ${ }^{4}$ The Hong Kong Polytechnic University \\ ${ }^{5}$ Auburn University
}

May 26, 2021

\begin{abstract}
Quantitative structure-property relationship (QSPR) studies based on deep neural networks (DNN) are receiving increasing attention due to their excellent performances. A systematic methodology coupling multiple machine learning technologies is proposed to solve vital problems including applicability domain and prediction uncertainty in DNN-based QSPRs. Key features are rapidly extracted from plentiful but chaotic descriptors by principal component analysis (PCA) and kernel PCA. Then, a detailed applicability domain $(\mathrm{AD})$ is defined by K-means algorithm to avoid unreliable predictions and discover its potential impact on uncertainty. Moreover, prediction uncertainty is analyzed with dropout-embedded DNN by thousands of independent tests to assess the reliability of predictions. The prediction of flashpoint temperature is employed as a case study demonstrating that the model accuracy is remarkably improved comparing with the referenced model. More importantly, the proposed methodology breaks through difficulties in analyzing the uncertainty of DNN-based QSPRs and presents an AD correlated with the uncertainty.
\end{abstract}

\section{Hosted file}

2021.5.19_wen.docx available at https://authorea.com/users/415953/articles/523705-asystematic-dnn-based-qspr-modeling-methodology-for-rapid-and-reliable-prediction-onflashpoints-of-chemicals 